\title{
The development of urban rail vehicle bogie virtual maintenance system Cang Song
}

\author{
Guidaojiaotong Polytechnic Institute, Shenyang 110023, China
}

\author{
Keyword: Virtual overhaul; Teaching System; bogie; Human-Computer Interaction
}

\begin{abstract}
According to structural characteristics and repair process of Urban Mass Transit Train bogies, A method of designing of virtual overhaul Teaching System is put forward combining 3D bogie modeling, virtual scene created and Human-Computer Interaction technology, that is Pro/E as a tool for $3 \mathrm{~d}$ assembly models, Used Visual $\mathrm{C}++2005$, OSG and Qt build up the virtual environment., Used Google SketchUp modeling the bogie overhaul workshop leading-in the virtual assembly system.. Introduced how to achieve the mutuality of the user to the virtual environment. and the bogie in the system. Shenyang urban mass transit train CCDZ50 bogies virtual overhaul was carried out due to those technologies above and is will use in the training of students in Shenyang Railway Polytechnic and maintenance workers in Shenyang Metro Operation Corporation.
\end{abstract}

\section{Introduction}

Shenyang metro vehicle adopts CCDZ50 type bogie, There are two squirrel cage asynchronous motors installed on the axle of the bogie, The structure is very complicated.urban rail vehicle bogie virtual maintenance system (hereinafter referred to as the maintenance system)The simulation is done by the software, you can directly presents the bogie structure, and reappear the whole maintenance process vividly. When the system is complete, on the one hand, there will be a good maintenance base in shenyang subway application requirements. On the other hand, it also can be used for urban rail vehicles professional teaching needs, improve the teaching quality at the same time, through testing to further consolidate the basic knowledge of students, improve student experiment skills.

\section{The production plan of Bogie virtual maintenance system}

Maintenance system mainly includes four parts, respectively is: model display system, bogie structure analysis system, database management system and 3D virtual maintenance system. The Bogie model and maintenance base scene model in the system using *. Osg format, using Pro/E modeling software to make 3D model, through the model import module converts 3D model to *. Osg format, as a system of geometric data model.Database management system used to store and display product maintenance hierarchy model, data management system at the same time, the system selects the SQL Server 2000 as the database management system.3D virtual maintenance system is to realize the product maintenance simulation, The virtual environment is established by OSG, vc ++2005 and Windows XP, Virtual maintenance was conducted in the virtual environment.

\section{The structure of the system and functional description}

System is divided into a master control module and four function modules, function modules are: virtual environment model module, operation module, database management module and system interface moduleThe four modules of the system are controlled by the same main module, system module structure as shown in figure 1.The main function of each module are as follows: 


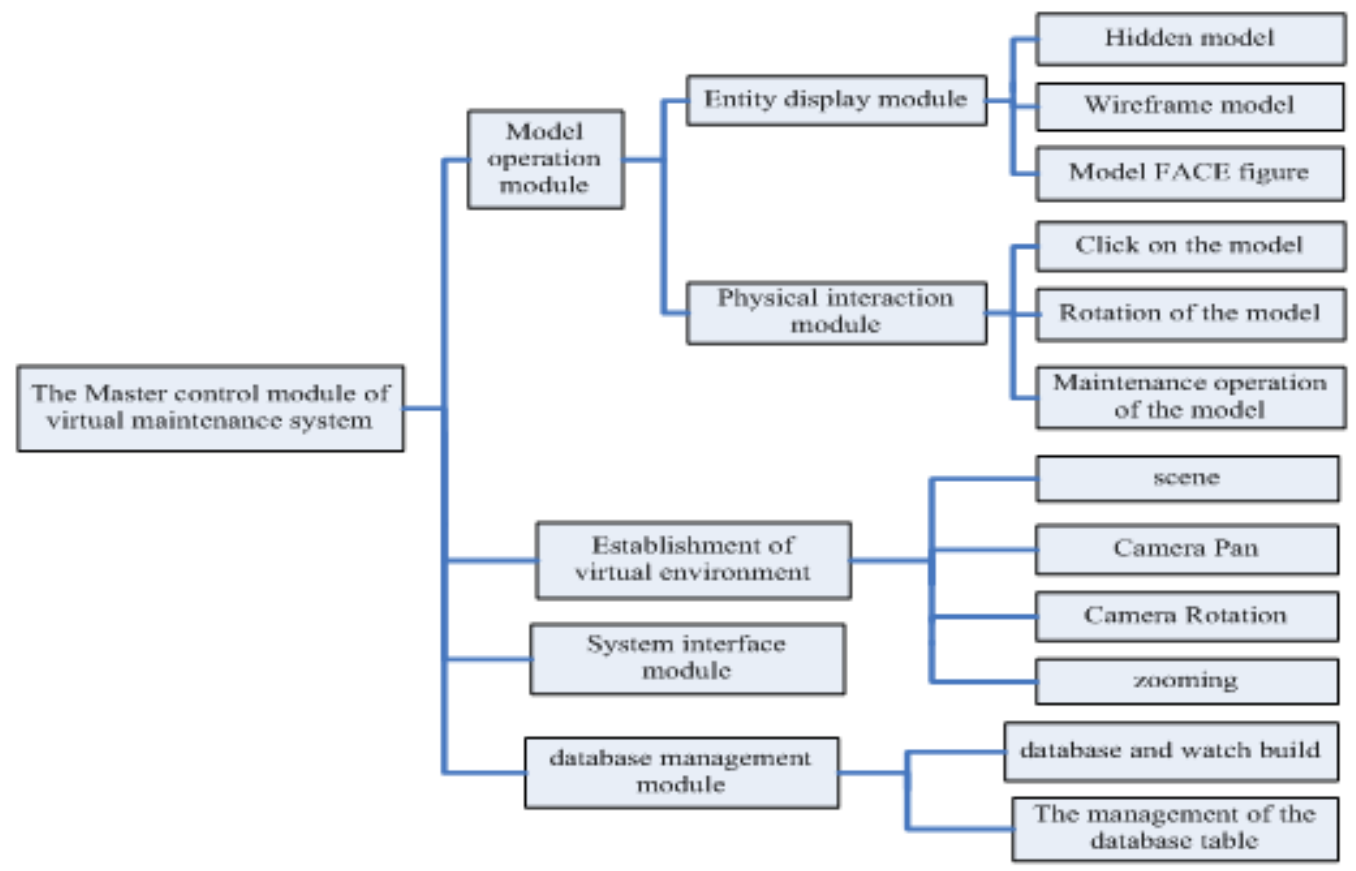

Fig.1 System module structure

(1)The system master control module, coordinate and control the operation of the following four modules.You can easily implement different functions in a program.

(2) Database management module: used for all data management system.In addition, according to the product model for database structure design, the system information is stored in the database with object-oriented technology, and the implementation model display and handle the result of the data in the database management and maintenance and other related functions.

(3) virtual environment to establish module: it is through the establishment of the graphical display of OSG viewport, is used for displaying the virtual maintenance environment, and includes the camera viewport related action, can from different angles, enlarged or reduced to watch objects, it also can change the background color and illumination.

(4) Model operation modules: operation object model in virtual environment, it is the key of virtual maintenance module, click through the module to complete the operation object, such as rotation, drag and drop function; On the other hand, can use entity face or wireframes observed objects.

(5) system interface module: by Qt writing, is the window of the system directly to the user.User interface for system operation through the system, all function modules of front-end display container that is.

\section{Create user interface application}

User interface is an important content of maintenance system, the user operating system are directly with the system interface, the system interface should include the function keys and virtual environment for virtual maintenance that operation maintenance system needed.After entering the system, users can use the mouse and function keys to click on the button on the interface to complete the corresponding instructions. The system choose Windows XP operating system with ve ++ and Qt 2005 created a combination of system operation interface.

According to the Qt for components, design three kinds of system related components: system main interface components, maintenance viewport components and functional components. Components, functional components including menu button component, component list component structure, the status bar, etc.Communication cooperation relationship between them is shown in figure 2 . 


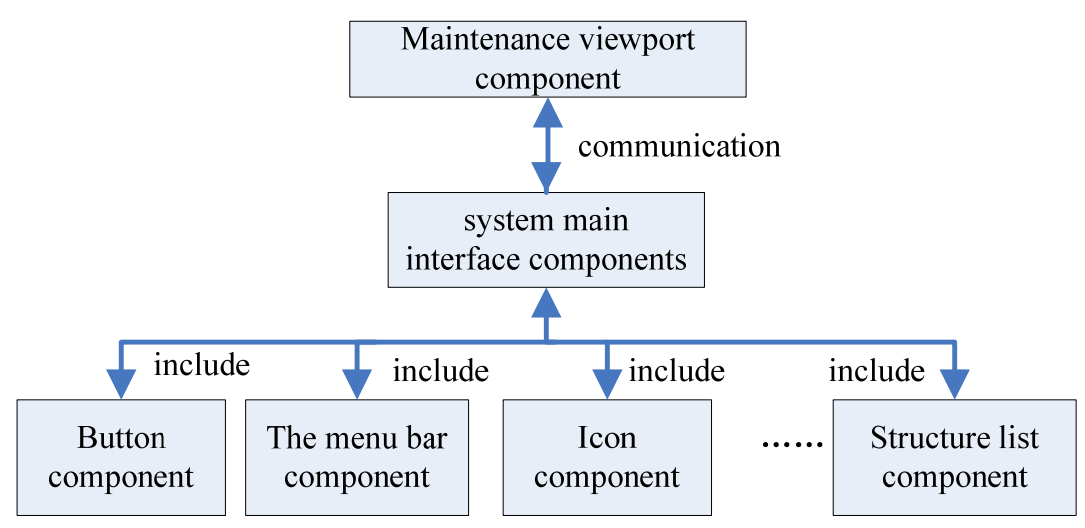

Fig.2 Communication between the system components

In the process of design, To separate the system main interface and virtual maintenance environment viewport that used for human-computer interaction. Respectively design the main interface components and component maintenance environment. The interface of virtual maintenance teaching system that Created by Qt as shown in figure 3.

\section{The system implementation and application example}

According to the introduction of system structure and each function module, the comprehensive effect of the main interface of virtual maintenance teaching system is established as shown in figure 3 , Access interface is divided into three parts, the top menu bar; The left is to repair parts name display area;Right is used to display $3 \mathrm{D}$ entity maintenance environment, all maintenance process will be realized in this environment and display;

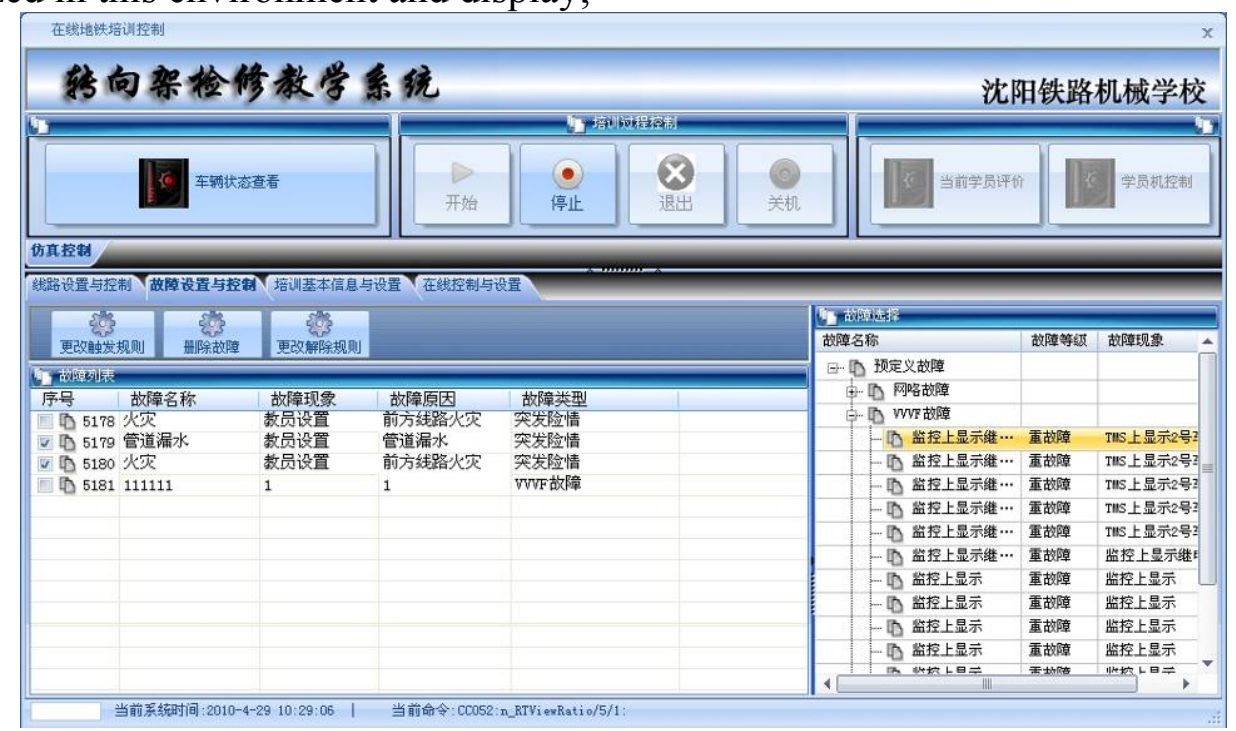

Fig.3 The virtual overhaul system interface

The interface of virtual maintenance repair in system as shown in figure 4.When need maintenance on a certain part, only need to click in the tree list, interface overhaul environment on the right side of the corresponding module will be displayed in the form of highlighting, and other modules will become translucent form to highlight the selected module.As shown in figure 4 is selected in air spring module display effect of the system. 


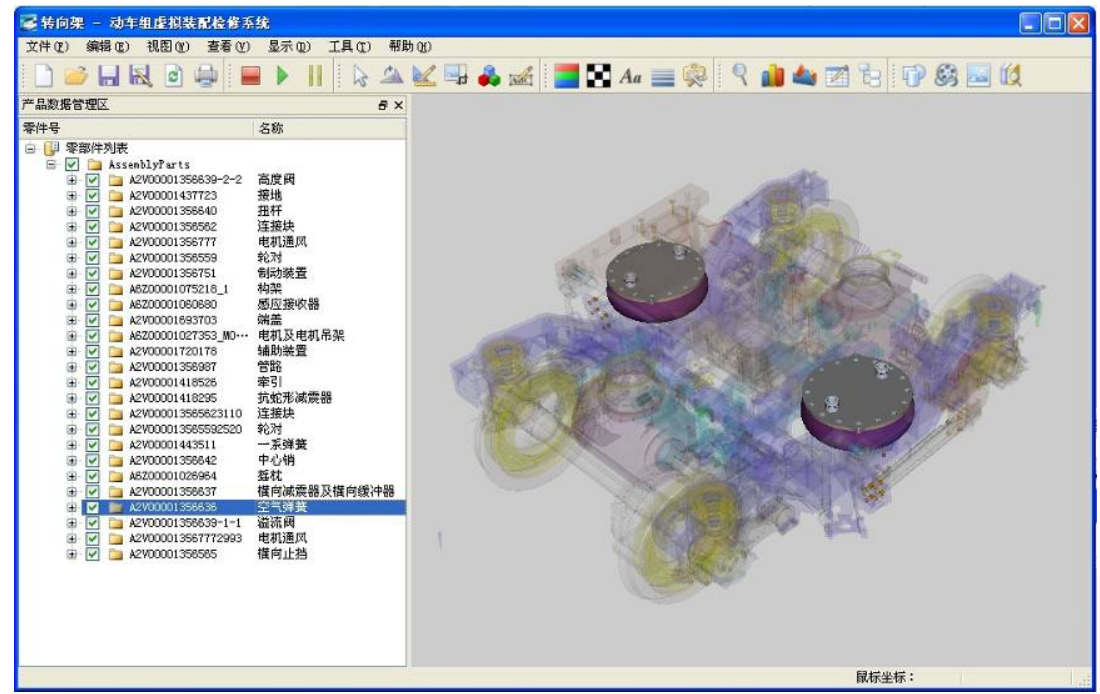

Fig.4 virtual overhaul interface

\section{Conclusion}

This paper presents a bogie maintenance teaching system design scheme and expounds the working principle of the system. Based on the above technology, urban rail vehicle bogie maintenance teaching system is designed.The teaching system structure is reasonable, function complete, cost-effective, The teaching system structure is reasonable, function complete, cost-effective, will be applied in the subway workers and school students' education training. Its design concept is not only suitable for urban rail transit, can also be used for vehicles, ships and aircraft maintenance system design for reference.

\section{References}

[1]Ma Lin,Lu Chuan. Study on virtual maintenance technology [J].Journal of Computer-Aided Design \& Computer Graphics, 2005,17(12):2729-2733 